\title{
BMJ Open Endoscopic polypectomy performed in clinic for chronic rhinosinusitis with nasal polyps: study protocol for the EPIC multicentre randomised controlled trial
}

Shaun Kilty, ${ }^{1,2}$ Kednapa Thavorn, ${ }^{1,3,4}$ Arif Janjua, ${ }^{5}$ John Lee, ${ }^{6}$ Kristian MacDonald, ${ }^{7}$ Eric Meen, ${ }^{8}$ Damian Micomonaco, ${ }^{9}$ Brian Rotenberg, ${ }^{10}$ Leigh J Sowerby, ${ }^{10}$ Marc Tewfik, ${ }^{11}$ Susan Adams, ${ }^{12}$ Hubert Frenette, ${ }^{12}$ Andrea Lasso (D) , Dean A Fergusson ${ }^{1,4,13}$

To cite: Kilty S, Thavorn K, Janjua A, et al. Endoscopic polypectomy performed in clinic for chronic rhinosinusitis with nasal polyps: study protocol for the EPIC multicentre randomised controlled trial. BMJ Open 2020;10:e042413. doi:10.1136/ bmjopen-2020-042413

- Prepublication history for this paper is available online. To view these files, please visit the journal online (http://dx.doi. org/10.1136/bmjopen-2020042413).

Received 03 July 2020 Revised 27 October 2020 Accepted 10 November 2020

A Check for updates

(C) Author(s) (or their employer(s)) 2020. Re-use permitted under CC BY-NC. No commercial re-use. See rights and permissions. Published by BMJ.

For numbered affiliations see end of article.

Correspondence to

Dr Shaun Kilty; skilty@toh.ca

\section{ABSTRACT}

Introduction Chronic rhinosinusitis (CRS) is common, with a Canadian prevalence of $5 \%$, and associated with significant morbidity. Understandably, CRS impairs workplace productivity but that productivity substantially increases following surgical treatment. CRS with nasal polyps (CRSwNP), the most common type of CRS, is usually treated with a combination of medications and endoscopic sinus surgery (ESS). Historically, surgical treatment has only been performed in the operating room at a cost of about $\$ C 3500$. However, recent studies have shown that a de-escalated procedure, endoscopic polypectomy performed in clinic (EPIC), can provide an improvement in patient symptoms to levels equal to those for ESS. Moreover, EPIC has additional proposed advantages including shorter recovery time, significantly lower cost to the healthcare system and shorter wait time for the patient. There is currently insufficient evidence to draw conclusions about the superiority of polypectomy or ESS for the management of CRSwNP.

Methods and analysis We designed a multicentre, openlabel, randomised controlled trial to evaluate whether EPIC was non-inferior to the current clinical standard, ESS for the treatment of CRSwNP. The primary outcome is the Sinonasal Outcome Test-22 score measured at baseline and at 3 months after surgery. Other outcomes include peak nasal inspiratory flow, quality of life measured by the EuroQoL 5 Dimensions 5 Levels questionnaire and work impairment using the Work Productivity and Activity Impairment Questionnaire.

We aim to recruit 140 patients from sites across Canada. Participants will be randomly assigned to EPIC or ESS and followed up for 3 months in clinic after the procedure. Additionally, participants will enter a 5-year long-term follow-up period.

Ethics and dissemination This study was approved by the Ottawa Health Sciences Network Research Ethics Board for all sites in Ontario, Canada (study number CT00801). Sites located outside of Ontario obtained approval from their local/institutional research ethics board.
Strengths and limitations of this study

This study is powered to determine if symptom improvement provided by in-clinic polypectomy is noninferior to that provided by endoscopic sinus surgery performed in the operating room in patients with chronic rhinosinusitis with nasal polyps.

- In this study, participants are randomly assigned to either in-clinic polypectomy or endoscopic sinus surgery thus reducing the influence of selection bias.

- This study is essentially limited to patients with large nasal polyps whose main clinical symptom is nasal obstruction.

- Participants will be followed via mailed questionnaires for 5 years to determine the length of duration of symptom improvement.

Trial registration number NCT02975310.

\section{INTRODUCTION}

Chronic rhinosinusitis (CRS) is a common problem affecting up to $5 \%$ of the Canadian population. ${ }^{1}$ It is a chronic disease that is associated with significant morbidity. ${ }^{2-4}$ It has a health state utility value that is equivalent to moderate asthma and end-stage renal disease requiring haemodialysis. ${ }^{5}$ Early studies indicate that CRS also leads affected individuals to be less productive in their workplace and that a patient's productivity increases substantially following surgical treatment. ${ }^{6-8}$ Similarly, although an affected patient's health-related quality of life is significantly impaired with CRS, this too has been shown to improve greatly with surgical treatment. ${ }^{5}$ Therefore, although CRS has a very significant impact on an individual's quality of life and their work productivity, in general, surgical treatment 
for CRS has been demonstrated to profoundly modify the impact of this disease on their symptoms and the disease's overall impact on the affected individual.

Endoscopic sinus surgery (ESS), the current gold standard surgical treatment for CRS with nasal polyps (CRSwNP), is normally completed with patients under general anaesthetic in an operating room. It involves the removal of any nasal polyps and the opening of affected paranasal sinuses. A systematic review by Cochrane concluded that 'there is currently no available evidence to support either nasal polypectomy or more extensive sinus clearance as a superior surgical modality in the treatment of chronic rhinosinusitis with polyps'. It recommended that randomised controlled trials be performed to determine if 'more extensive sinus dissection offers any advantage over endoscopic polypectomy alone'. ${ }^{9}$

ESS is performed in an operating room, thus there is a significant cost assigned to this procedure. A publication using a time-driven activity-based costing approach has demonstrated the institutional cost for this procedure to be at least $\$$ C3500. ${ }^{10}$ Given the prevalence of CRS and that one-fifth of patients will need another surgery within 5 years of the first procedure, ${ }^{11}$ the cost associated with surgical treatment for this disease is significant. Although the exact cost for the treatment of patients with CRS in Canada is not known, in the USA the total direct cost associated with CRS was estimated to be up to US\$64 billion in 2011. ${ }^{12}$ One expects the extrapolated costs in Canada for the management of this disease to also be elevated given similar disease prevalence. ${ }^{13}$ There is a clear need to evaluate cost-effective innovative ideas for the effective management of CRS.

A prior pilot study has evaluated one such potential innovation. ${ }^{14}$ To summarise, endoscopic polypectomy performed in clinic (EPIC) using topical and local anaesthesia can safely be performed with excellent patient pain control and, most importantly, providing significant symptom relief for a subset of patients with CRSwNP. This study has also demonstrated that the EPIC procedure had the potential for sizeable cost savings in comparison to the current standard ESS procedure. Similarly, in a case-control study of patients receiving EPIC or ESS, all demonstrated an equal disease-specific improvement in quality of life regardless of procedure ${ }^{1516}$ A recent health economic modelling study demonstrated that EPIC is a cost-saving option and potentially provides at least equivalent quality-adjusted life years (QALY) compared with ESS making it an attractive treatment option for the patients managed with EPIC. ${ }^{17}$ Given CRSwNP is a lifelong chronic condition and that, on average, patients require more than one surgery in their lifetime, these cost savings are additive. ${ }^{11}$ With the average wait time across Ontario, Canada, for ESS being about 6 months, ${ }^{18}$ EPIC offers patients a potentially shorter wait time to attain a surgical therapy and improve their quality of life. Therefore, EPIC may offer an alternative treatment for some patients with CRSwNP. We proposed a randomised controlled trial comparing the non-inferiority of EPIC to
ESS. If EPIC is non-inferior, our trial will allow surgeons to choose what is likely a less costly procedure for their patients but one that has similar efficacy and provides at least equal disease-specific improvements in quality of life. If EPIC is found inferior to ESS, the trial would provide level 1 evidence for ESS.

\section{METHODS AND ANALYSIS}

\section{Study design and intervention}

The study is a multicentre, open-label, randomised controlled trial evaluating whether EPIC is non-inferior to classic ESS in the operating room under general anaesthesia for the treatment of patients with CRSwNP. This study will be performed according to the Declaration of Helsinki, the Tri-Council Policy Statement 2, applicable provincial privacy legislation and Good Clinical Practice guidelines. This multicentre study started on 11 April 2017 and it is planned to end on 30 September 2023.

The experimental intervention is EPIC where nasal polyps are removed using a microdebrider under local (1\% lidocaine with 1:200000 epinephrine) and equal parts of topical anaesthetic (4\% lidocaine) with the topical vasoconstrictor epinephrine $(1: 1000)$ in the outpatient clinic. When possible, anaesthesia may include endoscopic injection of local anaesthetic into the lateral nasal wall. Only nasal polyps will be removed during EPIC, there will be no uncinate/bulla/ethmoid dissection/removal. After the procedure is completed, patients are observed in the clinic for 15-20 min or until any bleeding has stopped. Patients are not prescribed any medication for analgesia after EPIC; they are instructed to use acetaminophen if needed. The participant will be discharged home from the clinic following their procedure. Every procedure will be completed with the participant seated or supine in a reverse Trendelenburg (head in an elevated position). Having the head elevated has been demonstrated to decrease any bleeding associated with sinus surgery. ${ }^{19}$

The control intervention is ESS, a minimally invasive procedure that is the current standard that involves polypectomy with a microdebrider, ethmoidectomy, as well as sinus ostia enlargement of the affected sinuses performed in the operating room under general anaesthesia. Prior to ESS, each participant will undergo a routine evaluation at the institution's preoperative clinic for surgery education, physical evaluation and preoperative testing as necessary. At the time of surgery, general anaesthesia and airway control will be conducted as is customary for the anaesthesia staff of the participating institution. Local anaesthetic is used in every ESS case, primarily for the vasoconstrictive effects of the epinephrine that accompanies the lidocaine. Nonetheless, lidocaine is useful as this may decrease the possibility of pain central sensitisation, or the wind-up phenomenon, while also decreasing general anaesthetic needs. ESS will be performed following standard procedures; the extent of the surgery is determined at the discretion of each surgeon but 
includes complete polypectomy, ethmoidectomy and the opening of the ostia for all affected paranasal sinuses. After the surgery is complete, the participant will then be monitored in the postoperative anaesthetic care unit and then moved back to the surgical day care unit where they remain until they are ready for discharge home according to the institution's established practices. The institution's normal protocol for discharging patients after ESS will be followed. This includes the management of some participants who may require overnight admission to hospital following ESS due to underlying conditions (eg, obstructive sleep apnoea syndrome). Following ESS, patients are generally prescribed a small amount of narcotic medication (to be used only if needed), as per the operating surgeon's choice, that will last the patient $24-48$ hours at most. Every procedure will be completed with the participant's head in reverse Trendelenburg position.

All participants will receive a 5-day course of oral steroids immediately prior to their procedure as this therapy has been shown to decrease bleeding associated with polyp removal, ${ }^{20}$ and this treatment has been strongly endorsed in the literature for patients with CRSwNP undergoing surgical treatment. ${ }^{21}$

Daily topical steroids, the mainstay of treatment for CRS, will be stopped the day prior to the treatment intervention and restarted after the first follow-up is complete for all participants. Antibiotics will not be used preoperatively; however, if pus is evident at the time of the procedure, patients in both groups can be prescribed antibiotics postoperatively.

\section{Patient and public involvement}

Two patient representatives were invited to comment on the study design and were consulted in order to include patient-relevant outcomes in the design of the study. Patient representatives will be involved in the interpretation and dissemination of results.

\section{Eligibility criteria}

We will include adult participants (18 years and older) with a diagnosis of CRS with polyps requiring surgical treatment after having been treated with medical therapy as designated by the Canadian clinical practice guidelines for acute and chronic sinusitis. ${ }^{22}$ Participants will be required to have bilateral nasal polyps of grade $\geq 2$ on each side as determined by the Lildholdt scale score ${ }^{23}$ measured by nasal endoscopy at the screening visit. Participants must have a nasal blockage score greater than or equal to 2 on the Sinonasal Outcome Test-22 (SNOT-22); participants must also have an American Society of Anesthesiologists physical status 3 classification or less ${ }^{24}$ and must be able to provide informed consent.

We will exclude patients with a facial pain rated $>2$ on SNOT-22 at screening, participants with polyps large enough to cause external deformity and those whose nasal anatomy prevents appropriate scoring of nasal polyps (eg, severe septal deviation). Pregnant or breastfeeding women will be excluded. Participants with known history of Churg-Strauss syndrome, primary ciliary dyskinesia, or vasculitis (eg, granulomatosis with polyangiitis) as well as those with immunodeficiency, cystic fibrosis, allergic fungal sinusitis and aspirin exacerbated respiratory disease will be excluded. Participants allergic to any of the study medications or who are unable to undergo an awake procedure will be excluded.

\section{Study outcomes}

Primary outcome

The goal of this study is to evaluate the non-inferiority of EPIC versus ESS for the treatment of CRS with polyps (CRSwNP) for improvement of the symptoms of CRSwNP. The primary outcome measure for the symptoms of CRS with polyps is the SNOT-22 score measured at enrolment and 3 months after treatment. The trial is designed and powered to make this determination. The SNOT-22 score will also be measured at 6 months and at 1, 2, 3, 4 and 5 years after the index procedure. See table 1 for details on short-term and long-term follow-up procedures.

\section{Secondary outcomes}

A number of secondary outcomes are being used for this trial. Peak nasal inspiratory flow (PNIF) is an objective measurement of nasal inspiratory airflow. The improvement in nasal obstruction provided by each of the treatments at 3 months after treatment is being determined using a nasal inspiratory flow-meter. Patient satisfaction with anaesthesia for the treatment procedure is being evaluated with the Iowa Satisfaction with Anesthesia Scale (ISAS) after the procedure, on the same day it is done. It is a reliable and validated tool that evaluates patient satisfaction with procedural anaesthesia. ${ }^{25} 26$ Participant impairment of work productivity related to CRS is being evaluated prior to treatment and 3 months following treatment using the Work Productivity and Activity Impairment Questionnaire (WPAI). The WPAI is a validated six-item questionnaire developed as a patientreported assessment of absenteeism, presenteeism and impairment of daily activities due to a general or specific health problem (SHP) ${ }^{27}$ In this study, the SHP version of the questionnaire (the Work Productivity and Activity Impairment-Specific Health Problem) is being used as our disease of interest is CRS. The EuroQoL 5 Dimensions 5 Levels (EQ-5D-5L) is a standardised and validated measure that is being used to describe the health status of participants prior to and following either treatment at 3 months. ${ }^{28}$ This information will be used for economic analyses being completed with this trial. Individual health resource consumption is being collected regularly using the Participant Health Resource Consumption Survey (PHRCS); this instrument inquires about the health resource consumption related to CRS in the 90 days prior to the day of completion. It specifically asks about the use of (INCS) intra-nasal corticosteroids, oral corticosteroids and antibiotics, and visits to family doctor, ear, nose and throat or emergency room related to CRSwNP. This instrument also asks about additional surgical procedures 


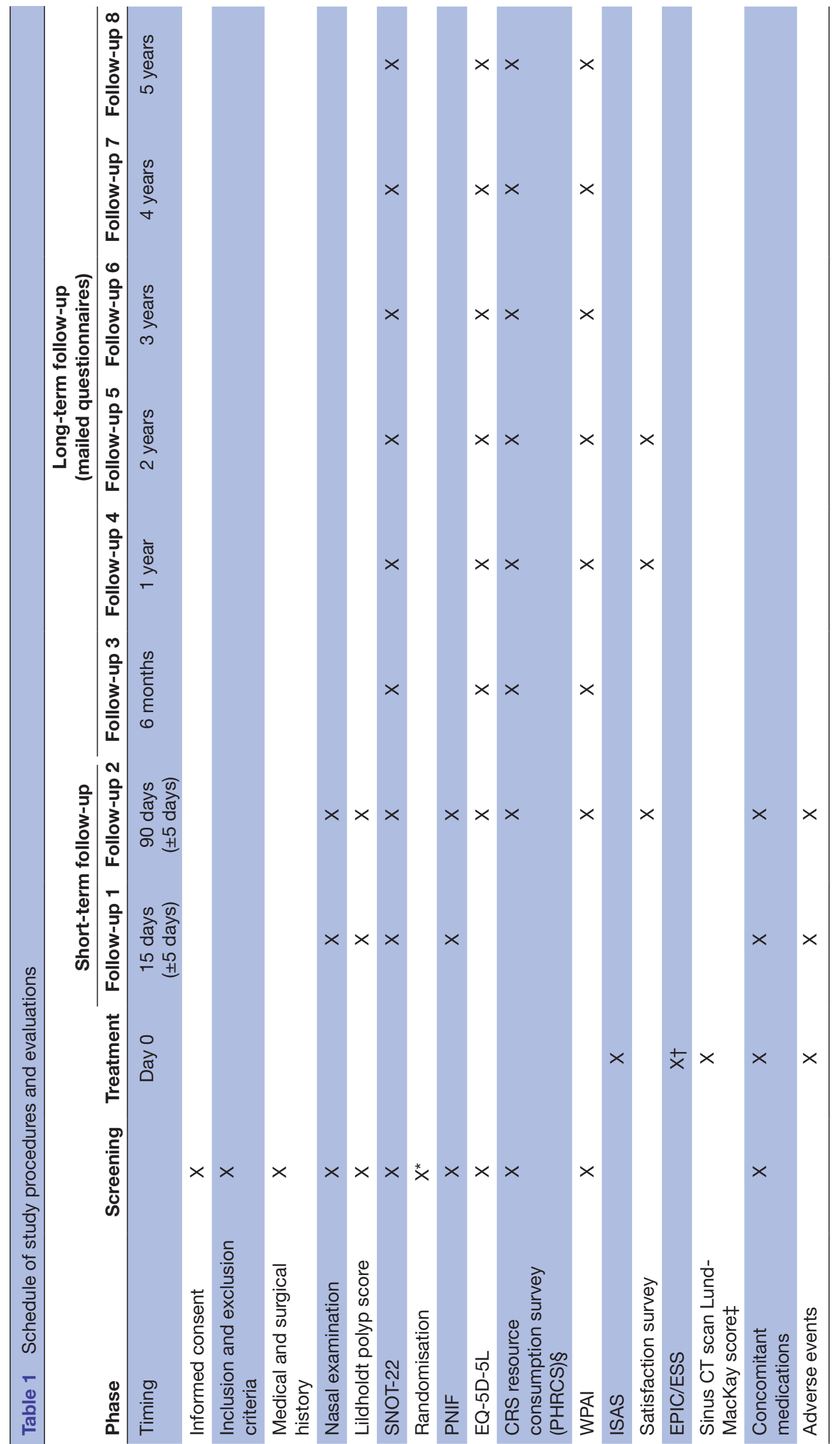

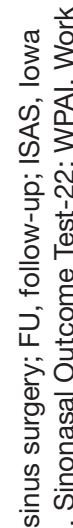

m.

马्य

흐응

0
0

出

产

ह ฮ.

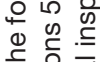

政

$\circ$ ब

당

은 농

离

งิ

这药

은 응

ه

잉

षं

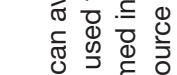

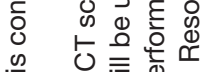

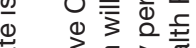

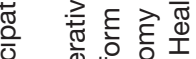

志

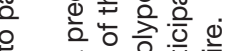

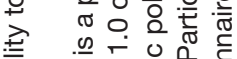

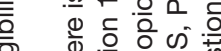

产

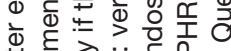

के

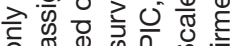

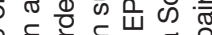

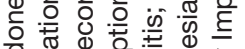

용

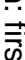

웅

$\frac{\bar{\sigma}}{\bar{D}}$

is

하

$\vec{\omega}$

홍.

$\frac{0}{8}$

กิ

ì

$\stackrel{+}{\oplus}$

인

N

प్

$\frac{10}{3}$

Nิ

뭉

흥

ㅇำ

$\overrightarrow{\overrightarrow{0}}$

沯

함

ه

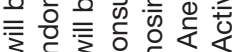

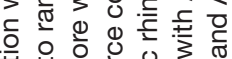

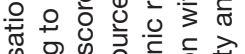

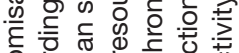

흠

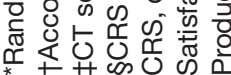


for CRSwNP since the index study procedure; this information will permit cost estimation for health resources used by patients with CRS and contribute to the costeffectiveness analysis. Last, all adverse events related to either surgical treatment will be recorded.

\section{Tertiary outcomes}

Endoscopic inflammation of the nose and paranasal sinuses after treatment will be evaluated using the LundKennedy Scale at the two post-treatment follow-up assessments. Satisfaction with each procedure will be evaluated using a visual analogue scale satisfaction survey.

The clinical tertiary outcomes of this study are only measured during the short-term follow-up period as the management of CRSwNP is primarily based on patient symptomatology.

\section{Sample size}

Our primary outcome, the SNOT-22 score, is a validated and reliable tool for assessing symptoms of CRS with polyps. ${ }^{29}$ Considering the primary outcome of SNOT-22 score, and based on an analysis of covariance (ANCOVA) test of the group differences in SNOT-22 scores, we calculated the minimum necessary sample size with the following assumptions: equal effects of EPIC and ESS; a margin of non-inferiority of 8.9 points, which is equal to the minimally clinically important difference of the SNOT-22 score; an SD of 20 points on SNOT-22 score; a $95 \%$ one-sided confidence limit on the difference in scores; and $80 \%$ power. Using a non-inferiority test of the null hypothesis that EPIC is superior to ESS by more than the margin of non-inferiority (8.9 points on the SNOT-22 score), a total of about 63 people would be required for enrolment in each arm of the study. Accounting for $10 \%$ dropouts and cross-overs from each arm of the trial, using the method of Lachin, ${ }^{30}$ an adjusted study size of 140 patients will be required. We selected a non-inferiority margin of 8.9 as it has been demonstrated to be the minimally important difference for the SNOT-22, that is, a change of less than 8.9 points cannot be perceived by a patient to be a clinically important improvement.

\section{Randomisation, allocation and data protection}

Eligible participants will be randomly assigned in a 1:1 fashion to either the experimental (EPIC) or the control group (ESS) using a web-based central randomisation to protect against selection bias. A permuted blocked randomisation method will be used to allocate eligible patients. An independent biostatistician will generate the randomisation scheme based on instructions from the study statistician. The randomisation process will consist of a computer-generated random listing of the treatment allocations in variable permuted blocks of 4 and 6 . The central web-based randomisation system is a programme that will ask a few questions to confirm eligibility and patient characteristics. The system will have backup in the form of a statistician and designate at the coordinating centre. Only the study statistician and designate at the coordinating centre will have knowledge of the randomisation codes. The process of central randomisation by the coordinating centre will help ensure that the randomisation scheme is not known by any study physicians at any site, thus eliminating any chance of manipulating treatment allocation. Furthermore, centralised randomisation allows for a master list to be constructed which will leave no doubt about who has and who has not been entered into the trial.

Blinding is not possible in this trial as both the investigator and the patient will be aware of the assigned treatment. To mitigate bias, study questionnaires and tests (e.g. SNOT-22, EQ-5D-5L, PNIF) will be administered by research assistants at the beginning of the study visit and scores will not be available to the surgeon investigators. The statistician performing the data analysis will be blinded as to what intervention was performed for each group.

\section{Participant timeline}

Study outcomes and timing of assessment are shown in table 1. After the screening visit, for both experimental (EPIC) and control (ESS) groups, the day the treatment is completed will be considered to be time ' 0 '. Enrolled participants will be re-evaluated by the investigators after their procedure twice, which is the normal frequency of visits for postoperative care for patients who have undergone surgical treatment for CRSwNP. The first follow-up evaluation at 15 days ( \pm 5 days) following the procedure and the last will occur 90 days ( \pm 5 days) following the treatment. The last follow-up is chosen to be at 90 days given the primary outcome measure of interest is the SNOT-22 score. Three months after treatment, the SNOT-22 does not appear to change substantially with further follow-up. A landmark study completed by Hopkins $e t a l^{31}$ with a cohort of over 3000 patients who had undergone ESS for chronic sinusitis and who were followed for 36 months demonstrated that the SNOT-22 scores at 3 months following surgery were not different from those measured at 12 or 36 months. ${ }^{31}$ Therefore, the 3-month (90 days) follow-up has been specifically chosen for this study given the primary outcome measure being used is also the SNOT-22 score.

After the second follow-up visit, patients will enter a long-term follow-up period which will last 5 years. During this period, patients will receive yearly study questionnaires via mail. At this time, patients will also be asked if they have had additional surgeries for their CRSwNP. See table 1 for detailed description of timing of study procedures.

\section{Study status}

This multicentre study started on 11 April 2017 with six study sites across Canada. Currently, 73 patients have been enrolled in the study.

\section{Data analysis plan}

Baseline analyses

Baseline characteristics of patients in the two treatment arms will be assessed using frequency distributions and 
univariate descriptive statistics including measures of central tendency and dispersion. A second step will involve comparing all major baseline variables for each of the trials with independent t-tests (equivalent nonparametric procedures when standard assumptions are not met; ie, Wilcoxon rank-sum test) for continuous data and $\chi^{2}$ tests for categorical data.

\section{Principal analysis of primary outcome measure}

As this is an effectiveness trial, all statistical analyses will be based on an intention-to-treat approach. All enrolled participants will be analysed according to the intervention to which they were allocated, whether they received it or not. However, given our non-inferiority hypothesis and its susceptibility by way of dilution of effect by noncompliers in the intention-to-treat approach, we will also conduct a treatment received as per protocol analysis of the primary outcome measure.

An unadjusted mean difference in 3-month SNOT-22 scores with 95\% CIs will be calculated to ascertain whether EPIC is non-inferior to ESS (ie, assessing our 8.9 margin of non-inferiority). We will also compare 3-month SNOT-22 scores between patients allocated to EPIC and ESS using a Student's t-test or Wilcoxon rank-sum test depending on whether the SNOT-22 data are parametric or nonparametric. In addition, repeated ANCOVA models will be used to further elucidate the measure of effect while adjusting for possible confounding variables and baseline/follow-up SNOT-22 measurements. Covariates will be added to the models based on their clinical significance. Pairs of variables will be considered for inclusion into models if there is sufficient statistical evidence and the interaction has clinical rationale.

\section{Principal analysis of secondary outcome measures}

For each of our secondary outcomes EQ-5D-5L, PNIF, ISAS, WPAI and Lund-Kennedy Scale, we will conduct either Student's t-tests or Wilcoxon rank-sum tests based on normality assumptions. In addition, linear regression models will be used to further elucidate the measure of effect while adjusting for possible confounding variables.

An interim analysis will be performed once $60 \%$ of the participants have completed the 3-month follow-up visit.

We will also conduct a cost-utility analysis of EPIC compared with ESS alongside the trial that follows patients up to 90 days following the index procedure. At these follow-up times it will be determined what, if any, additional costs of care for CRS were incurred. Health utility scores will be measured using the EQ-5D-5L questionnaire. These utility values will be used to calculate a QALY based on a total area under the curve method. ${ }^{32}$

A net-benefit regression approach ${ }^{33}$ will be used to translate the differences in cost and quality of life of patients between EPIC and ESS into a common monetary term. Equation 1 shows the means of calculating the net monetary benefit, where ' $\lambda$ ' is the maximum willingness to pay per one QALY gained, ' $\Delta \mathrm{E}$ ' is the difference in QALY and ' $\Delta \mathrm{C}$ ' is the difference in cost.

$$
N M B=* \Delta E-\Delta C
$$

The use of regression will permit us to adjust for any remaining confounders and repeated measures of the trial data. Uncertainty in the analysis will be assessed by estimating $95 \%$ CI using a non-parametric bootstrapping method. The results of the uncertainty analysis will be also presented as a cost-effectiveness acceptability curve representing the probabilities that EPIC is cost-effective over the range of willingness to pay.

The regression method will also identify any major patient characteristics that drive differences in treatment effectiveness and/or cost. If there are any significant characteristics (in terms of statistical and quantitative significance) identified, we will stratify our analysis according to these patient characteristics to test the possibility that different treatment strategies are more favourable in certain patient cases.

\section{Safety reporting and study monitoring}

Adverse events will be evaluated at every clinic visit after screening. All serious adverse events should be reported to the coordinating centre within 24 hours of becoming aware of the event and follow-up information should be sent as soon as it becomes available.

A Data Safety Monitoring Board will be responsible for assuring that the study participants in the EPIC trial are not exposed to unnecessary or unreasonable risks and that the study is being conducted according to the highest and ethical standards.

The central study coordinator will perform site monitoring, verification of case report form data against source documentation on-site and remotely.

\section{Ethics and dissemination}

This study was approved by the Ottawa Health Sciences Network Research Ethics Board for all participating sites in Ontario, Canada (study number CTO0801). Sites located outside of Ontario obtained approval from their local/ institutional research ethics board before commencement of the study. The study is registered on the ClinicalTrials.gov website and study results will be available in the same registry once available. Results will be disseminated via peer-reviewed publication and presentation at relevant national and international conferences.

\section{Author affiliations}

${ }^{1}$ Clinical Epidemiology Program, Ottawa Hospital Research Institute, Ottawa, Ontario, Canada

${ }^{2}$ Department of Otolaryngology- Head \& Neck Surgery, University of Ottawa, Ottawa, Ontario, Canada

${ }^{3}$ ICES u0ttawa, Institute for Clinical Evaluative Sciences, Ottawa, Ontario, Canada ${ }^{4}$ School of Epidemiology and Public Health, University of Ottawa, Ottawa, Ontario, Canada

${ }^{5}$ Department of Surgery, Division of Otolaryngology-Head and Neck Surgery, The University of British Columbia, Vancouver, British Columbia, Canada

${ }^{6}$ Department of Otolaryngology-Head and Neck Surgery, Saint Michael's Hospital, Toronto, Ontario, Canada

${ }^{7}$ Department of Surgery, Dalhousie University, Halifax, Nova Scotia, Canada

${ }^{8}$ Department of Otolaryngology, University of Manitoba, Winnipeg, Manitoba, Canada 
${ }^{9}$ Department of Clinical Sciences, Northern Ontario School of Medicine, Thunder Bay, Ontario, Canada

${ }^{10}$ Department of Otolaryngology - Head and Neck Surgery, Western University,

London, Ontario, Canada

${ }^{11}$ Department of Otolaryngology-Head and Neck Surgery, McGill University,

Montreal, Quebec, Canada

${ }^{12}$ Patient Representative, Ottawa, Ontario, Canada

${ }^{13}$ Department of Medicine, Faculty of Medicine, University of Ottawa, Ottawa, Ontario, Canada

Contributors SK and DAF conceptualised the study. AJ, JL, KM, EM, DM, BR, LJS and MT provided expert support in rhinology and helped in the design of the final study protocol. KT conceptualised the health economic evaluation aspect of the protocol. SK is the principal investigator responsible for the overall conduct of the study. SK, AJ, JL, BR, LJS and MT are local principal investigators responsible for recruitment, clinical care and data collection. SA and HF are patient representatives who provided input on selection on relevant patient outcomes. AL helped in technical design of the protocol, and coordinated the ethics approval and collaboration among investigators from all institutions. SK wrote the manuscript and all authors reviewed and approved the final version of the manuscript.

Funding This work is supported by the Canadian Institutes of Health Research (CIHR; grant number PJT148734) and Medtronic Xomed through a PhysicianSponsored Research Grant (agreement number: A 1284455). The cost-effectiveness analysis is supported by a Health Research Grant (26 June 2017) from the PSI Foundation.

Disclaimer The funders did not have any input in the development of the protocol and neither will they have input in the analysis of the data.

Competing interests None declared.

Patient and public involvement Patients and/or the public were involved in the design, or conduct, or reporting, or dissemination plans of this research. Refer to the Methods section for further details.

Patient consent for publication Not required.

Provenance and peer review Not commissioned; externally peer reviewed.

Open access This is an open access article distributed in accordance with the Creative Commons Attribution Non Commercial (CC BY-NC 4.0) license, which permits others to distribute, remix, adapt, build upon this work non-commercially, and license their derivative works on different terms, provided the original work is properly cited, appropriate credit is given, any changes made indicated, and the use is non-commercial. See: http://creativecommons.org/licenses/by-nc/4.0/.

ORCID iD

Andrea Lasso http://orcid.org/0000-0002-9552-264X

\section{REFERENCES}

1 Chen Y, Dales R, Lin M. The epidemiology of chronic rhinosinusitis in Canadians. Laryngoscope 2003;113:1199-205.

2 Kilty SJ, McDonald JT, Johnson S, et al. Socioeconomic status: a disease modifier of chronic rhinosinusitis? Rhinology 2011;49:533-7.

3 Gliklich RE, Metson R. The health impact of chronic sinusitis in patients seeking otolaryngologic care. Otolaryngol Head Neck Surg 1995;113:104-9.

4 Macdonald KI, McNally JD, Massoud E. The health and resource utilization of Canadians with chronic rhinosinusitis. Laryngoscope 2009;119:184-9.

5 Soler ZM, Wittenberg E, Schlosser RJ, et al. Health state utility values in patients undergoing endoscopic sinus surgery. Laryngoscope 2011;121:2672-8.

6 Stankiewicz J, Tami T, Truitt T, et al. Impact of chronic rhinosinusitis on work productivity through one-year follow-up after balloon dilation of the ethmoid infundibulum. Int Forum Allergy Rhinol 2011;1:38-45.

7 Stankiewicz J, Truitt T, Atkins J, et al. Two-Year results: transantral balloon dilation of the ethmoid infundibulum. Int Forum Allergy Rhinol 2012;2:199-206.

8 Rudmik L, Smith TL, Schlosser RJ, et al. Productivity costs in patients with refractory chronic rhinosinusitis. Laryngoscope 2014;124:2007-12.
9 Sharma R, Lakhani R, Rimmer J, et al. Surgical interventions for chronic rhinosinusitis with nasal polyps. Cochrane Database Syst Rev 2014;11:CD006990.

10 Au J, Rudmik L. Cost of outpatient endoscopic sinus surgery from the perspective of the Canadian government: a time-driven activitybased costing approach. Int Forum Allergy Rhinol 2013;3:748-54.

11 Hopkins C, Slack R, Lund V, et al. Long-Term outcomes from the English national comparative audit of surgery for nasal polyposis and chronic rhinosinusitis. Laryngoscope 2009;119:2459-65.

12 Caulley L, Thavorn K, Rudmik L, et al. Direct costs of adult chronic rhinosinusitis by using 4 methods of estimation: results of the US medical expenditure panel survey. J Allergy Clin Immunol 2015;136:1517-22

13 Tan BK, Kern RC, Schleimer RP, et al. Chronic rhinosinusitis: the unrecognized epidemic. Am J Respir Crit Care Med 2013;188:1275-7.

14 Kilty SJ. In-clinic endoscopic polypectomy for chronic rhinosinusitis with polyps: a treatment strategy pilot study in ten adults. Clin Otolaryngol 2015;40:281-4

15 Kilty SJ, Lasso A, Mfuna-Endam L, et al. Case-Control study of endoscopic polypectomy in clinic (EPIC) versus endoscopic sinus surgery for chronic rhinosinusitis with polyps. Rhinology 2018;56:155-7.

16 Caulley L, Lasso A, Rudmik L, et al. Pre-Treatment SNOT-22 score predicts response to endoscopic polypectomy in clinic (EPIC) our experience in 30 adults. Clin Otolaryngol 2017;42:732-4.

17 Kumar S, Thavorn K, van Katwyk S, et al. Cost-Effectiveness of endoscopic polypectomy in clinic compared to endoscopic sinus surgery: a modelling study. Clin Otolaryngol 2020;45:477-85.

18 OMoHaL-T C. Ontario wait times, 2010.

19 Gan EC, Habib A-RR, Rajwani A, et al. Five-degree, 10-degree, and 20 -degree reverse Trendelenburg position during functional endoscopic sinus surgery: a double-blind randomized controlled trial. Int Forum Allergy Rhinol 2014;4:61-8

20 Wright ED, Agrawal S. Impact of perioperative systemic steroids on surgical outcomes in patients with chronic rhinosinusitis with polyposis: evaluation with the novel perioperative sinus endoscopy (pose) scoring system. Laryngoscope 2007;117:1-28.

21 Poetker DM, Jakubowski LA, Lal D, et al. Oral corticosteroids in the management of adult chronic rhinosinusitis with and without nasal polyps: an evidence-based review with recommendations. Int Forum Allergy Rhinol 2013;3:104-20.

22 Desrosiers M, Evans GA, Keith PK, et al. Canadian clinical practice guidelines for acute and chronic rhinosinusitis. J Otolaryngol Head Neck Surg 2011;40 Suppl 2:S99-193.

23 Côté DWJ, Wright E. Objectiveoutcomes in endoscopic sinus surgery. In: Advances in endoscopic surgery. InTech, 2011. http:// www.intechopen.com/books/advances-in-endoscopic-surgery/ objective-outcomes-in-endoscopic-sinussurgery

24 (ASA) ASoA. Asa physical status classification system, 2014. Available: https://www.asahq.org/resources/clinical-information/asaphysical-status-classification-system [Accessed Oct 2014]

25 Dexter F, Candiotti KA. Multicenter assessment of the lowa satisfaction with anesthesia scale, an instrument that measures patient satisfaction with monitored anesthesia care. Anesth Analg 2011;113:364-8.

26 Dexter F, Aker J, Wright WA. Development of a measure of patient satisfaction with monitored anesthesia care: the lowa satisfaction with anesthesia scale. Anesthesiology 1997;87:865-73.

27 Reilly MC, Zbrozek AS, Dukes EM. The validity and reproducibility of a work productivity and activity impairment instrument. Pharmacoeconomics 1993:4:353-65.

28 EuroQol Group. EuroQol--a new facility for the measurement of health-related quality of life. Health Policy 1990;16:199-208.

29 Hopkins C, Gillett S, Slack R, et al. Psychometric validity of the 22item sinonasal outcome test. Clin Otolaryngol 2009;34:447-54.

30 Lachin JM. Introduction to sample size determination and power analysis for clinical trials. Control Clin Trials 1981;2:93-113.

31 Hopkins C, Browne JP, Slack R, et al. The National comparative audit of surgery for nasal polyposis and chronic rhinosinusitis. Clin Otolaryngol 2006;31:390-8.

32 Manca A, Hawkins N, Sculpher MJ. Estimating mean QALYs in trialbased cost-effectiveness analysis: the importance of controlling for baseline utility. Health Econ 2005;14:487-96.

33 Hoch JS, Briggs AH, Willan AR, et al. Something blue: a framework for the marriage of health econometrics and cost-effectiveness analysis. Health Econ 2002;11:415-30. 